\title{
1.3 Die Auseinandersetzung mit der Sowjetunion und der Spaltungsprozess in der amerikanischen politischen Linken
}

Indessen bedurfte es nicht erst der totalitären Mächtekoalition von 1939, um sich in den Vereinigten Staaten mit dem kulturellen und politischen Totalitarismus auseinanderzusetzen und - was an dieser Stelle insbesondere von Bedeutung ist - ein kritisches Bild des Sowjetstaates zu zeichnen. Aus zwei Richtungen näherte man sich bereits im Verlauf der späten 1930er-Jahre der »russischen Frage«: Einerseits waren es insbesondere eine Reihe renommierter New Yorker (Links-)Intellektueller, die sich mehr und mehr von der kommunistischen Idee und vom stalinistischen Herrschaftsregime abwandten und sich $\mathrm{zu}$ Exponenten eines intellektuellen politischen Antikommunismus wandelten, andererseits machten einflussreiche Vertreter der »Roosevelt-Administration « persönliche Erfahrungen mit den konkreten innenpolitischen Verhältnissen in der Sowjetunion, die ihnen dabei halfen, in den kommenden Jahren zu einer realistischen Analyse und nüchternen Einschätzung der Moskauer Außenpolitik bzw. des Charakters des sowjetischen Staates zu kommen.

Unter dem Eindruck von Stalins Terrorpolitik Mitte der 1930er-Jahre kam es in den Vereinigten Staaten zur Auseinandersetzung mit dem Stalinismus, die einen grundsätzlichen Zerfallsprozess der KPUSA und ihr nahestehenden »fellow-traveller«-Organisationen - die über ein weitgefächertes kulturelles und politisches Netz die öffentliche Meinung über die Sowjetunion in den Vereinigten Staaten nicht unwesentlich beeinflussten - und letztlich die Polarisierung der amerikanischen (Links-)Intellektuellen in Gang setzte. Zur Galionsfigur der antistalinistischen »radical intellectuals« avancierte neben dem prominenten Philosophen John Dewey der aus dem politisch einflussreichen Kreis der New York Jewish Intellectuals kommende Sidney Hook.

Hook hatte wesentlichen Anteil am Zustandekommen der vom American Commitee for the Defense of Leon Trotsky im März 1937 ins Leben gerufenen Kommission zur Untersuchung der Moskauer Schauprozesse. Unter der Leitung von John Dewey, der sich unter anderem auf die Mitarbeit bekannter Intellektueller wie James Burnham, John Dos Passos, Max Eastman, James T. Farrell, Dwight Macdonald, Mary McCarthy, Norman Thomas, Reinhold Niebuhr und Lionel Trilling verlassen konnte, wurde der terroristische Herrschaftscharakter des sowjetischen Regimes unter Beweis gestellt. Stalins Politik zwang nicht nur Hunderttausende von Unschuldigen in den Lagern, Zwangsarbeit zu vollrichten, sondern die willkürlich formulierten Anklagepunkte gegen Trotzki unter anderem sowie das Entstehen der Prozessurteile in Moskau zeigten vor allem eines: das wahre Wesen des Sowjetkommunismus. Dewey griff die linksliberalen Intellektuellen an, die die Zustände in der Sowjetunion kritiklos hinnahmen und zum Terror schwiegen. Er verurteilte das stalinistische Herrschaftssystem, »das von Anfang an auf Gewalt gegründet war und über kurz oder lang von seinen selbstentfesselten Destruktivkräften verzehrt würde « ${ }^{35}$.

In diesem Zusammenhang ging es Hook in einer Auseinandersetzung mit Bruce Bliven, dem Herausgeber der den linksliberalen Gruppen engverbundenen Zeitschrift

35 Bernd Greiner, Die Morgenthau-Legende. Zur Geschichte eines umstrittenen Plans, Hamburg 1995, S. 68. Zum »American Commitee for the Defense of Leon Trotsky« siehe Alan M. Wald, The New York Intellectuals. The Rise and Decline of the Anti-Stalinist Left from the 1930 s to the 1980s, Chapel Hill/ London 1987, S. 128-156. 
New Republic, primär darum, seine Kritik an einer politischen Haltung zu äußern, die zwar gegenüber sozialen und politischen Ungerechtigkeiten in den Vereinigten Staaten, Deutschland und Spanien sensibel reagierte und ihren Unmut äußerte, indes die Verhältnisse in der Sowjetunion totschwiegen. ${ }^{36}$ Im Verlauf ihrer Auseinandersetzung mit dem stalinistischen Herrschaftsregime nahmen Dewey und Hook mehr und mehr eine totalitarismustheoretische Grundhaltung in Bezug auf die bedrohlichen weltpolitischen Entwicklungen in den späten 1930er-Jahren ein, die sie durch die existenzielle politische Frontstellung von liberaldemokratischen und totalitären Staaten gekennzeichnet sahen. Am 15. Mai 1939 riefen sie in New York das Committee for Cultural Freedom ins Leben und veröffentlichten am 22. Mai 1939 in der Zeitschrift The Nation und am 29. Mai in der New York Post ein für hohes Aufsehen sorgendes Manifest, aus dem die kulturellen und politischen Grundüberzeugungen der neuerrichteten Institution zum Ausdruck kamen. Insgesamt 96 Unterzeichner $^{37}$ formulierten darin eine "gleichermaßen von individualistischen und sozialistisch-egalitären Prinzipien getragenen Kritik« an den totalitären Diktaturen in Deutschland, Italien, Sowjetrussland, Japan und Spanien, plädierten zudem aber auch für eine permanente Kritik an möglichen regressiven Gesellschaftsentwicklungen in den Vereinigten Staaten.

Die Reaktion aus dem Umfeld der Kommunistischen Partei bzw. der »RooseveltLinken« (Bernd Greiner) ließ nicht lange auf sich warten. In den meisten Publikationen wurde das Benutzen des Totalitarismusparadigmas als unzulässige Gleichsetzung von Faschismus und Kommunismus abgelehnt und auf die besonderen Differenzen insistiert. Die am meisten diskutierte Veröffentlichung erschien ebenfalls in der Zeitschrift The Nation, die den Spaltungsprozess innerhalb der politischen Linken in den Vereinigten Staaten vertiefen sollte. In dem von 400 Intellektuellen - unter anderem Waldo Frank, Granville Hicks, Max Lerner und Harry A. Ward - unterzeichneten Leserbrief An alle aktiven Unterstützer von Freiheit und Frieden wurde die Distanz zur antistalinistischen Linken und zum Committee for Cultural Freedom offenkundig, indem die angeblich bereits realisierten kulturellen und wissenschaftlichen Freiheiten in der Sowjetunion gepriesen wurden. Der stalinistische Terror wurde keineswegs geleugnet, aber als notwendiges Übel auf dem Weg zur nächsthöheren Gesellschaftsstufe betrachtet. Überhaupt sei die Sowjetunion, so die Unterzeichner, der alleinige Hort des Friedens und insofern prädestiniert, den Nationalsozialismus zu bekämpfen. Als ein historischer »Treppenwitz « musste es erscheinen, dass der Leserbrief am 26. August 1939 erschien, also drei Tage nach dem völlig überraschenden Abschluss des Hitler-Stalin-Paktes. ${ }^{38}$

Der von den allermeisten Zeitgenossen als Überraschung und als Schock empfundene deutsch-sowjetische Nichtangriffspakt sorgte zum einen für einen Desillu-

36 Vgl. Cornelie Kunkat, Sidney Hook. Intellektueller zwischen Marxismus und Pragmatismus, Frankfurt a. M./New York 2000, S. $204 \mathrm{f}$.

37 Hochgeschwender (Freiheit in der Offensive?, S. 99, Anm. 167) weist darauf hin, dass der überwiegende Teil der Unterzeichner Professoren aus dem universitären Bereich waren, allerdings nur wenige der zukünftigen antikommunistischen Intellektuellen darunter waren. Er nennt u. a. Sherwood Anderson, Rudolf Carnap, Merle Curti, Max Eastman, Sol Levitas, John Dos Passos, Willi Schlamm, Arthur Schlesinger jr., Norman Thomas und Dorothy Thompson.

38 Siehe Greiner, Die Morgenthau-Legende, S. 72. 
sionierungsprozess in Teilen der politischen Linken in den Vereinigten Staaten ${ }^{39}$ und verstärkte dort den antistalinistischen bzw. antikommunistischen Kurswechsel, der in den wichtigen linken New Yorker Zeitschriften wie Partisan Review und New Leader entsprechend unterstützt wurde. Zum anderen war die imperialistisch-machtpolitische Komplizenschaft der beiden diktatorischen Regime der auslösende Faktor, die systematische Analyse der Gemeinsamkeiten von Stalinismus und Nationalsozialismus voranzutreiben und den Begriff der totalitären Herrschaft in der Wissenschaft für beide Herrschaftssysteme zu verwenden. ${ }^{40}$ Festzuhalten bleibt, dass sich in den Vereinigten Staaten im Verlauf der späten 1930er-Jahre in bestimmten Gruppen der politischen Linken und bei einigen ihnen nahestehenden Intellektuellen sowie der amerikanischen Öffentlichkeit die Wahrnehmungsperspektive in Bezug auf die Sowjetunion sukzessive veränderte. Der Sowjetkommunismus unter Stalin wurde in Analogie zum Nationalsozialismus als totalitäres Herrschaftsregime klassifiziert, das unter anderem durch die Attribute exzessiver Terror in der Innenpolitik und imperiale Weltherrschaftsziele in der Außenpolitik gekennzeichnet sei und sich durch einen antidemokratischen Furor auszeichnete. Erst das »Unternehmen Barbarossa«, d.h. der deutsche Angriff auf die Sowjetunion im Juni 1941, und die sich herauskristallisierende politische Allianz zwischen den westlichen Staaten und Stalin bis zum Ende des Zweiten Weltkrieges 1945 ließen es inopportun erscheinen, den Bündnispartner als einen totalitären Staat zu betrachten. Gleichzeitig bedeutete die notwendig gewordene Anti-Hitler-Koalition eine vorübergehende Stillstellung der weltpolitischen Polarität Demokratie versus Totalitarismus. ${ }^{41}$

39 Vgl. hierzu zum Beispiel Wolfgang Leonhard, Der Schock des Hitler-Stalin-Paktes. Erinnerungen aus der Sowjetunion, Westeuropa und USA, Freiburg i. Br. 1986, S. 165-172, und auf S. 182-186 die Erinnerungen des ehemaligen Mitglieds der KPUSA Granville Hicks.

40 Es sei daran erinnert, dass wenige Monate nach dem Hitler-Stalin-Pakt im November 1939 in Philadelphia die erste wissenschaftliche Konferenz stattfand, auf der die Teilnehmer explizit die Totalitarismustheorie als Analyserahmen für den Faschismus bzw. Nationalsozialismus und Sowjetkommunismus akzeptierten. Siehe die entsprechenden Ausführungen im Einleitungskapitel.

41 Krawtschenko sprach im Nachwort seines 1946 in New York erschienenen und zum Bestseller avancierenden autobiografischen Renegatenbuches I Chose Freedom diesen Sachverhalt an, dass er trotz des Wissens um die wirklichen Zustände des sogenannten kommunistischen »Polizeistaates« und der täglichen persönlichen Vernichtungsdrohung in den Vereinigten Staaten durch den allmächtigen sowjetischen Geheimdienstapparat von Lawrenti Berija die weltweiten Gefahren durch die sowjetkommunistische Diktatur nicht der Öffentlichkeit mitteilte. Krawtschenko konzedierte: »Die Wichtigkeit der militärischen Zusammenarbeit zwischen den westlichen Demokratien und der totalitären Sowjetunion [im Kampf gegen den Nationalsozialismus] legte mir große Zurückhaltung auf. Ich nahm sie willig an. Die Notwendigkeit des gemeinsamen Sieges ging allem anderen vor. Jetzt aber, da der Krieg siegreich beendigt ist, halte ich es nicht nur für berechtigt, sondern auch für meine ausdrückliche Pflicht, alles ehrlich und so wirksam wie möglich zu sagen. Deshalb dieses Buch.« (Zit. n. Victor A. Kravchenko, Ich wählte die Freiheit. Das Private und politische Leben eines Sowjetbeamten, Hamburg o. J., S. 625) Auf die politische Bedeutung des Autors und des Buches komme ich in Kap. III zurück. 